\title{
Dispersed fs-FWM for Investigations of Low Frequency Vibrations of Transient Species in Combustion
}

\author{
Gregor Knopp*, Peter P. Radi, and Thomas Gerber
}

\begin{abstract}
Understanding the role of peroxides in combustion, in atmospheric and in surface science involves investigations on a molecular level, at which energy transfers and rearrangements of the nuclei dictate the chemistry. Due to their high reactivity they appear as unstable transient species, which impedes detailed spectroscopic investigations. Low frequency modes, especially internal and hindered rotations with energies less than $200 \mathrm{~cm}^{-1}$ play an important role for the determination of molecular energies and reaction rates. Unfortunately, these motions are in general difficult to address. Femtosecond four-wave mixing (fs-FWM) is a powerful tool that allows for investigations of ground state dynamics of molecules. The high peak-power output of fs-lasers, facilitates the excitation of weak Raman coherences. Results from the combustion relevant di-tert-butyl peroxide molecule are exemplified.
\end{abstract}

Keywords: Four-wave mixing · Low frequency vibrations · Peroxides · Time- and frequency resolved

\section{Introduction}

Peroxides constitute a class of molecules that are of fundamental importance in the understanding of combustion processes. ${ }^{[1]}$ Already the onset of hydrocarbon oxidation has to occur via the chemical attack of oxygen on the fuel (e.g. alkane) molecules forming $\mathrm{HOO}^{*}$ and alkyl $\left(\mathrm{R}^{\circ}\right)$ radicals. The fate of these radicals depends on temperature and pressure conditions of the combustive environment. At high temperatures e.g. exothermic reactions start producing alkenes and di-hydrogen peroxides $(\mathrm{HOOH})$, which subsequently dissociate into ${ }^{\circ} \mathrm{OH}$ radicals, while at low temperatures the molecular kinetics and dynamics of alkyl peroxy radicals (ROO*) significantly determine the reaction progress. Due to the high reactivity, alkoxy ( $\mathrm{RO}^{\circ}$ ) and alkyl peroxy radicals are unstable transient species at high temperatures. This fragility hampers a sufficient production of peroxides for spectroscopic investigations and as consequence ROO' radicals are mostly not well characterized. Di-alkyl peroxides (ROOR) provide a higher stability, and therefore are safer

\footnotetext{
${ }^{*}$ Correspondence: Dr. G. Knopp

Paul Scherrer Institute

General Energy Department

$\mathrm{CH}-5232$ Villigen

Tel.: +41563104108

Fax: + 41563102166

E-mail: gregor.knopp@psi.ch
}

in handling and more practical for primary spectroscopic investigations. Nevertheless, they are quite thermally and photolytically sensitive due to a facile breaking of the oxygen-oxygen bond. This unimolecular dissociation mainly produces $\mathrm{RO}^{\circ}$, which then, via chain propagating reactions, potentially forms other radicals. For this reason di-alkyl peroxides are usually used as oxygen-containing additives in hydrocarbon combustion. ${ }^{22}$ Tertiary dialkyl peroxides, however, are prevalently better suited to generate free radicals in commercial applications because they are less susceptible to radical-induced decompositions and are often more efficient radical generators than primary or secondary ones. ${ }^{[3]}$ In this respect, di-tert-butyl-peroxide (di-tert-BOO), for example, is a well known fuel additive, that has been applied for ignition control in diesel engines, in which the ignition process accelerates due to the increased presence of $\mathrm{RO}^{\circ}$ radicals, produced from the di-tert-BOO dissociation. ${ }^{[2]}$

Understanding the role of peroxides in combustion, in atmospheric and in surface science involves investigations on a molecular level, at which energy transfers and rearrangements of the nuclei dictate the chemistry. Rotations, vibrations, conformation and fragmentation can occur on sub-ns timescales and adequate monitoring requires ultrafast spectroscopy. Pumpprobe techniques with temporal resolution of $\sim 100$ fs are state of the art. However, because of a poor energy resolution due to time-bandwidth limitation, many approaches lack specificity. In this respect, methods that involve combined time-fre- quency detection strategies are superior to pure time-resolved techniques in measuring the dynamics with an increased specification. ${ }^{[4]}$

\section{Method}

Femtosecond four-wave mixing (fsFWM) is a powerful tool that allows investigation of ground state dynamics of molecules. ${ }^{[5-10]}$ It has been widely used to study ro-vibrational motions, ${ }^{[9,11]}$ couplings, ${ }^{[12,13]}$ and energy transfer ${ }^{[14-16]}$ at ultrafast molecular timescales. The measurement concept of time-resolved FWM involves a material polarization that is induced by a pair of laser fields and subsequently probed by a third one. In the frequency domain several FWM methods rely on Raman-photon excitations and experiments depend on a sufficient spectral resolution to identify the characteristic energy shifts. In the time domain, high spectral accuracy can be alternatively achieved by Fourier transformation of the data, where the technical resolving power is essentially limited by the applied time delays. ${ }^{[9,17]}$

Extended molecular systems exhibit a complex spectroscopy and time-consuming long scanning procedures require stable, well defined samples. In this respect, the unspecific excitation by short, thus energetically broad laser pulses involves a rather difficult analysis of the measured signals. The question whether frequency- or timeresolved approaches are the methods of choice, is not only a matter of optimizing the pulse parameters towards smallest achievable bandwidth or shortest duration. 
Though both domains are interconnected by Fourier transformation, the desired information is only achieved by measurements sampling both regimes. ${ }^{[18-20]}$ Such 'twodimensional', time-frequency approaches contain combined information about the chemical systems of investigation. ${ }^{[21]}$

Raman-type excitations are adequate processes for investigating ro-vibrational modes in the ground electronic state and fs-coherent anti-Stokes Raman scattering $[7,8,16,22-26]$ has been proven to be a convenient method. However, approaching low energetic vibrations, the impulsive fsRaman excitation involves spectrally overlapping pump and Stokes pulses and the method becomes degenerate (fs-DFWM). In time domain fs-DFWM measurements, the coherent excitation of many energetically close lying rotational transitions leads to characteristic recurring signals. Relaxation and dephasing processes due to collisions can impede the observation of rotational recurrences at such long time delays. In these cases, the most prominent rotational feature in the fs-DFWM signal is observed within the first few ps. Though the determination of rotational constants by fitting the early time response of the molecule can provide a one- to two-digit accuracy, the short time delay of a few ps only implies a serious constraint in spectral resolution, hampering a sufficient specification by Fourier transformation.

Coherent heterodyne techniques are well known for the enhancement of weak signals due to spectral interference with a local oscillator-field. The extra field needs to be in phase with the signal and the measurement depends linearly on the induced polarization $S_{h e t} \propto \varepsilon_{L O} P^{(3)}$ compared to the quadratic dependence considering homodyne detection $S_{\text {hom }} \propto\left|P^{(3)}\right|^{2}$. Spectral interferometry (SI) per se is a heterodyne technique in which two temporally separated light pulses are dispersed in a spectrometer (and therefore spread in time) and interfere. In analogy partial heterodyning occurs in dispersed fs-DFWM without implementation of an extra field, if parts of the signal that are generated at different times enter the spectrometer simultaneously and interfere. Low frequency modes may visualize via mixed terms. Inherent sources for 'self'-heterodyning, thus for the generation of interfering extra fields in fs-FWM are possibly nonresonant contributions, sample ionization or constant polarization terms. ${ }^{[19,27-29]}$ An interfering field of approx. $\sim 1 \%$ of the signal intensity already can cause modulations depths of $\sim 40 \%$ in the spectra. ${ }^{30]}$ Similar to intentionally induced heterodyning, 'self'-heterodyning leverages the signals resulting in an improved analysis by using dispersed detection methods with the advantage that phase-stabilization issues are automatically fulfilled.

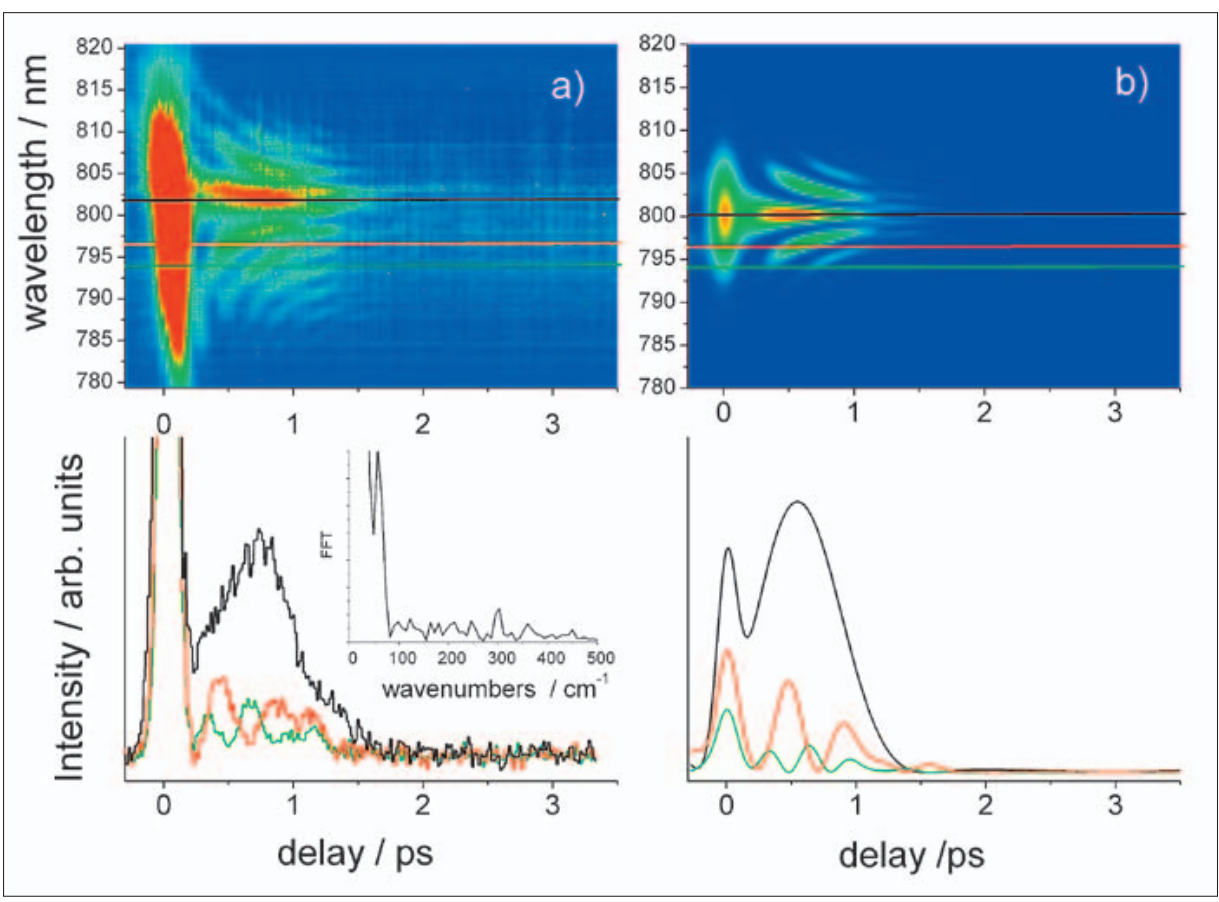

Fig. 1. a) Dispersed fs-DFWM signal from di-tert-BOO. b) Simulation, considering rotational transitions only. A sectional view at three detection wavelengths (black, red, green) and a Fourier transformation of the experiment at $802 \mathrm{~cm}^{-1}$ is depicted in the graphs below.

\section{Dispersed DFWM from Di-tert-BOO}

The general investigation of low frequency modes of transient species in the gas phase is certainly not restricted to one specific molecule only. Beside its importance in combustion, the given example of di-tert-BOO comprises a ro-vibrational complexity that is common to other substantially sized molecules and peroxides.

The molecular response from timefrequency resolved DFWM measurements of 20 mbar di-tert-butyl peroxide in the gas phase has been measured. We confine ourselves to a time-frequency analysis of electronically off-resonance fs-DFWM, using the $100 \mathrm{fs} / 800 \mathrm{~nm}$ output from a regenerative amplified Ti:Sapphire fs-laser system (CLARK CPL1000). The typical spectral bandwidth of the $800 \mathrm{~nm}$ laser pulses, derived from a FROG measurement is $\sim 170$ $\mathrm{cm}^{-1}$ (FWHM) providing a time bandwidth product of 0.52 and a 155 fs (FWHM) autocorrelation width. Adjacent to a nonresonant time zero contribution, the signal at early delays is mainly characterized by an at first view relatively unspecific peak of approx. one picosecond duration as depicted in Fig. 1a. Considering the rather small rotational constants of di-tert-BOO $\mathrm{A}_{0}=0.0246 \mathrm{~cm}^{-1}, \mathrm{~B}_{0}=0.02478 \mathrm{~cm}^{-1}, \mathrm{C}_{0}=$ $0.0751 \mathrm{~cm}^{-1}$, this feature is mainly caused by re- and dephasing of the Raman polarization, due to rotational motions with slow angular velocities.

The dispersed fs-DFWM signal resembles an interference pattern on the CCD detector as function of the delay $\tau$. Fig. 1a shows the signal intensity (color code: blue to red) as a function of the delay time and the detection wavelength $\left(\lambda_{\text {de }}\right)$ The observed hyperbolic curvatures become plausible by considering 'self heterodyning' of the signal. A simulation (Fig. 1b) of the observed intensity distribution could be realized by consideration of a strong interfering nonresonant signal contribution. Though the simulation in Fig. 1b does not include any low frequency vibrations it already displays the most significant signal modulations observed. Cuts at three distinct detection wavelengths are additionally displayed.

Approximately twelve low frequency modes ${ }^{[31]}$ of di-tert-BOO are possibly covered by the bandwidth of the 100 fs laser pulses. However, only a few Raman signals below $300 \mathrm{~cm}^{-1}$, assigned as the $\delta(\mathrm{OOC})$ at $243 \mathrm{~cm}^{-1}$, the $\tau\left(\mathrm{CH}_{3}\right)$ at $175 \mathrm{~cm}^{-1}$ and the $\delta\left(\right.$ OCC) at $290 \mathrm{~cm}^{-1}$ deformation and torsion vibrations of di-tert-BOO, have been observed. ${ }^{[32]}$ Hereby the $\delta(O O C)$ deformation at $243 \mathrm{~cm}^{-1}$ provides the strongest Raman activity with approx. $\sim 25 \%$ of the strength of the OO stretch at $862 \mathrm{~cm}^{-1}$. Though the bandwidth of the applied laser pulses is not sufficient to efficiently measure the $243 \mathrm{~cm}^{-1}$ deformation, a sectional view at the centre wavelength $(\sim 802 \mathrm{~nm})$ in Fig. 1 displays a fast oscillation on top of the rotational structure that corresponds to a frequency of $\sim 300$ $\mathrm{cm}^{-1}$ as can be seen in the power spectrum of the selected slice. The most prominent peak in the power spectrum is observed around $50 \mathrm{~cm}^{-1}$. Fourier transformation at each spectral position detuned by $\Delta\left[\mathrm{cm}^{-1}\right]$ 
from the probe centre wavelength yields a $2 \mathrm{D}$ plot of the measured signal as shown in Fig. 2. The intensity of the peak between 30 and $70 \mathrm{~cm}^{-1}$ is not uniformly spread over the probe pulse spectrum and characteristics within the bandwidth of the laser pulse at different detuning positions become visible. Simulation (rotations only) and experiment display a detailed structure in the twodimensional plane. Though the simulation considering rotational Raman transitions only predicts off-centre peaks at approx. 50 $\mathrm{cm}^{-1}$, the experimental results partially differ from the simulation and display a more detailed structure. This observed structure is most probably not only due to rotational features of the molecule. Low-frequency torsional vibration modes that turn into free rotations at excitation energies above their barriers can interfere with the rotational motions. Still, identification and specification of such low frequency modes is rather difficult. The accuracy of the observed peak positions along the wavenumber dimension that is limited by the resolution of the Fourier transformation is $\sim 8 \mathrm{~cm}^{-1}$. Measuring the signals till $16 \mathrm{ps}$ delay time increases the theoretical resolving power to $\sim 2 \mathrm{~cm}^{-1}$. Fig. 3 results from such a measurement and displays an even more detailed structure of the $50 \mathrm{~cm}^{-1}$ peak.

\section{Conclusion and Outlook}

Calculations $^{[31]}$ on a second order Møller-Plesset level of perturbation theory using a 6-31 G(d,p) basis set have proposed a low frequency torsion motion at $58 \mathrm{~cm}^{-1}$. At this stage of our data analysis it is too early to say whether such a $58 \mathrm{~cm}^{-1}$ peak, evident in the dispersed spectra, corresponds to this torsion motion. However, in contrast to purely homodyne detected DFWM, we expect to observe indications from the fundamental vibrations to appear at off-centre detection positions if partial 'self-heterodyning' occurs. In Fig. 2 and 3 several peaks appear detuned from the centre detection frequency and display bimodal structures. It seems as if a second rotational feature starts to emerge from $\sim 30 \mathrm{~cm}^{-1}$ onwards. Therefore confidence is given that the observed beating patterns yield information on torsion motions having a temporal evolution that is comparable to the rotational motion of the molecules. Investigations of low frequency modes of molecules by terahertz radiation are complementary approaches within the MUST program. Further, the optical phases of the involved laser pulses have a strong influence on the results that are possibly achieved from coherent measurement concepts based on interference. A detailed analysis of the phase influence and the development of possible coherent control

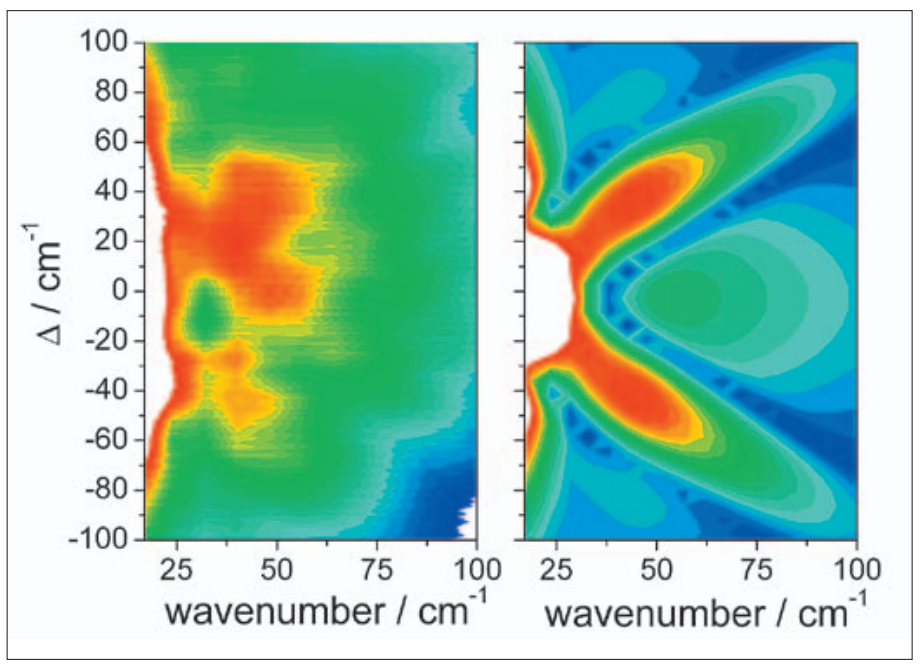

Fig. 2. Fourier transformation of the data given in Fig. 1 in respect to the delay time $\tau$. The ordinate displays the off-center wavenumber position $\Delta$, relative to the center wavenumber of the signal $(\Delta<0$ red shifted, $\Delta>0$ blue shifted).

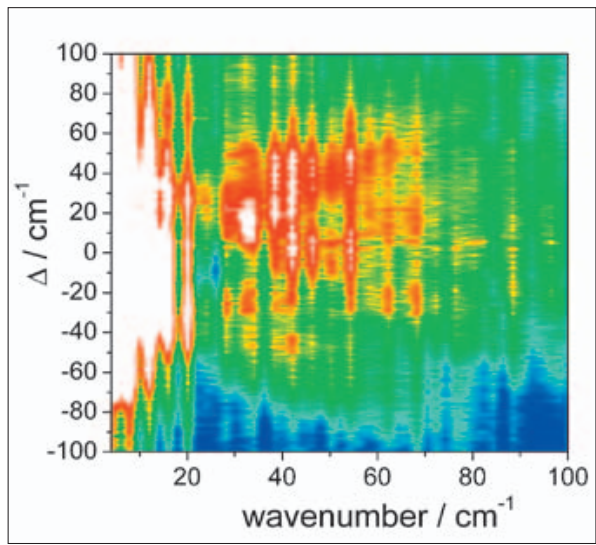

Fig. 3. Two-dimensional plot similar to Fig. 2 with a FFT resolution of $\sim 2 \mathrm{~cm}^{-1}$.

strategies towards the enhancement of low frequency motions will be compiled within the MUST framework.

\section{Received: March 8, 2011}

[1] C. A. Taatjes, N. Hansen, A. McIlroy, J. A. Miller, J. P. Senosiain, S. J. Klippenstein, F. Qi, L. Sheng, Y. Zhang, T. A. Cool, J. Wang, P. R. Westmoreland, M. E. Law, T. Kasper, K. KohseHoinghaus, Science 2005, 308, 1887.

[2] K. Tanaka, K. Yamada, T. Nakagawa, K. Kuchitsu, J. Mol. Spec. 1975, 54, 243.

[3] Y. T. Kenneth, W. F. Paul, K. C. L. Edward, J. Chem. Phys. 1977, 66, 3303.

[4] A. M. Walser, M. Meisinger, P. P. Radi, T. Gerber, G. Knopp, Phys. Chem. Chem. Phys. 2009, 8456.

[5] M. Dantus, V. V. Lozovoy, Chem. Rev. 2004, 104, 1813.

[6] V. V. Lozovoy, M. Comstock, I. Pastirk, M Dantus, in 'Femtochemistry and Femtobiology: Ultrafast Events in Molecular Science', Ed. M. M. Martin, J. T. Hynes, Elsevier, 2004, pp 33-36.

[7] M. Schmitt, G. Knopp, A. Materny, W. Kiefer, Chem. Phys. Lett. 1997, 280, 339.

[8] M. Schmitt, G. Knopp, A. Materny, W. Kiefer, Chem. Phys. Lett. 1997, $270,9$.

[9] H. M. Frey, P. Beaud, T. Gerber, B. Mischler, P. P. Radi, A. P. Tzannis, Appl. Phys. B 1999, B68, 735.

[10] A. M. Walser, P. Beaud, P. P. Radi, M. Tulej, T. Gerber, G. Knopp, J. Raman Spect. 2007, 38, 147.
[11] V. V. Matylitsky, W. Jarzeba, C. Riehn, B Brutschy, J. Raman Spect. 2002, 33, 877.

[12] D. M. Besemann, K. A. Meyer, J. C. Wright, J. Phys. Chem. B 2004, 108, 10493.

[13] M. A. Rickard, A. V. Pakoulev, K. Kornau, N. A Mathew, J. C. Wright, J. Phys. Chem. A 2006, 11384 and 3352.

[14] P. Beaud, T. Gerber, P. P. Radi, M. Tulej, G. Knopp, Chem. Phys. Lett. 2003, 373, 251.

[15] P. Beaud, G. Knopp, Chem. Phys. Lett. 2003, 371, 194.

[16] G. Knopp, P. Radi, M. Tulej, T. Gerber, P. Beaud, J. Chem. Phys. 2003, 118, 8223.

[17] T. Lang, M. Motzkus, H. M. Frey, P. Beaud, J. Chem. Phys. 2001, 115, 5418.

[18] J. C. Wright, Abstracts of Papers of the American Chemical Society 2005, 230, 366.

[19] A. Shalit, Y. Paskover, Y. Prior, Chem. Phys. Lett. 2008, 450, 408 .

[20] T. Hornung, J. C. Vaughan, T. Feurer, K. A Nelson, Opt. Lett. 2004, 29, 2052.

[21] R. P. Lucht, Science 2007, 316, 207.

[22] J. Faeder, I. Pinkas, G. Knopp, Y. Prior, D. J. Tannor, J. Chem. Phys. 2001, 115, 8440.

[23] G. Knopp, P. Beaud, P. Radi, M. Tulej, B Bougie, D. Cannavo, T. Gerber, J. Raman Spect. 2002, 33, 861 .

[24] G. Knopp, I. Pinkas, Y. Prior, J. Raman Spect. 2000, 31, 51 .

[25] I. Pinkas, G. Knopp, Y. Prior, J. Chem. Phys 2001, 115, 236.

[26] O. Rubner, M. Schmitt, G. Knopp, A. Materny, W. Kiefer, V. Engel, J. Phys. Chem. A 1998, 102, 9734 .

[27] V. G. Stavros, E. Harel, S. R. Leone, J. Chem. Phys. 2005, 122, 064301

[28] V. G. Stavros, S. R. Leone, Chem. Phys. Lett 2008, 452, 33

[29] T. Seideman, Phys. Rev. Lett. 1999, 83, 4971.

[30] R. P. Trebino, 'Frequency-resolved optical gating', Kluwer Academic Publishers, 2011.

[31] S. L. Khursan, V. L. Antonovsky, Russ. Chem. Bull. 2003, 52, 1312 .

[32] I. P. Zyat'kov, G. A. Pitsevich, A. P. Yuvchenko, L. Y. Ol'dekop, V. I. Gogolinskii, V. L. Antonovskii, D. I. Sagaidak, translated from Zhurnal Prikladnoi Spektroskopii 1988, 49, 271. 